Acta Crystallographica Section E

Structure Reports

Online

ISSN 1600-5368

\section{2,8-Dimethyl-10-p-tolyl-10H-phenoxa- phosphine}

\section{Thashree Marimuthu, Muhammad D. Bala* and Holger B. Friedrich}

School of Chemistry, University of KwaZulu-Natal, Westville Campus, Private Bag X54001, Durban 4000, South Africa

Correspondence e-mail: bala@ukzn.ac.za

Received 5 March 2009; accepted 17 March 2009

Key indicators: single-crystal X-ray study; $T=173 \mathrm{~K}$; mean $\sigma(\mathrm{C}-\mathrm{C})=0.002 \AA$; $R$ factor $=0.037 ; w R$ factor $=0.108 ;$ data-to-parameter ratio $=19.0$.

The title compound (systematic name: 3,6-dimethyl-10-p-tolyl9-oxa-10-phosphaanthracene), $\mathrm{C}_{21} \mathrm{H}_{19} \mathrm{OP}$, is a precursor for the preparation of a bidentate xanthene-based ligand, in which the dihedral angle between the toluene ring and the phenoxaphosphine ring system is $83.26(3)^{\circ}$. The geometry at the $\mathrm{P}$ atom is pyramidal, resulting in a longer $\mathrm{C}-\mathrm{P}$ bond length as compared to the two ring $\mathrm{C}-\mathrm{P}$ bonds.

\section{Related literature}

For related structures based on the xanthene backbone, see: Marimuthu et al. $(2008 a, b, c)$. For a related phenoxaphosphine compound, see: Mann et al. (1976). The title compound was synthesised by a modified literature method (Bronger et al., 2004). For other structurally related ligands, see: Levy et al. (1965); Seibold et al. (2008); Shau et al. (2002).<smiles>Cc1ccc(P2c3cc(C)ccc3Oc3ccc(C)cc32)cc1</smiles>

\section{Experimental}

Crystal data
$\mathrm{C}_{21} \mathrm{H}_{19} \mathrm{OP}$
$M_{r}=318.33$
Monoclinic, $P 2_{1} / c$
$a=10.9363(3) \AA$
$b=11.6323(3) \AA$
$c=14.0458(4) \AA$
$\beta=111.532(1)$

\section{Data collection}

Bruker APEXII CCD area-detector diffractometer

Absorption correction: none

29900 measured reflections

Refinement

$R\left[F^{2}>2 \sigma\left(F^{2}\right)\right]=0.037$

$w R\left(F^{2}\right)=0.108$

$S=1.07$

4013 reflections

$$
\begin{aligned}
& V=1662.13(8) \AA^{3} \\
& Z=4 \\
& \text { Mo } K \alpha \text { radiation } \\
& \mu=0.17 \mathrm{~mm}^{-1} \\
& T=173 \mathrm{~K} \\
& 0.51 \times 0.49 \times 0.48 \mathrm{~mm}
\end{aligned}
$$

4013 independent reflections 3507 reflections with $I>2 \sigma(I)$ $R_{\text {int }}=0.046$

Data collection: APEX2 (Bruker, 2005); cell refinement: SAINTPlus (Bruker, 2005); data reduction: SAINT-Plus; program(s) used to solve structure: SHELXTL (Sheldrick, 2008); program(s) used to refine structure: SHELXL97 (Sheldrick, 2008); molecular graphics: SHELXTL; software used to prepare material for publication: SHELXTL.

We thank Dr Manuel Fernandes for the data collection and acknowledge SASOL, THRIP and the University of KwaZulu-Natal for financial support.

Supplementary data and figures for this paper are available from the IUCr electronic archives (Reference: RZ2300).

\section{References}

Bronger, R. P. J., Bermon, J. P., Herwig, J., Kamer, P. C. J. \& van Leeuwen, P. W. N. M. (2004). Adv. Synth. Catal. 346, 789-799.

Bruker (2005). APEX2 and SAINT-Plus. Bruker AXS Inc., Madison, Wisconsin, USA.

Levy, J. B., Doak, G. O. \& Freedman, L. D. (1965). J. Org. Chem. 30, 660-661. Mann, F. G., Millar, I. T., Powell, M. \& Watkin, D. J. (1976). J. Chem. Soc. Perkin Trans. 2, pp. 1383-1384.

Marimuthu, T., Bala, M. D. \& Friedrich, H. B. (2008a). Acta Cryst. E64, o711. Marimuthu, T., Bala, M. D. \& Friedrich, H. B. (2008b). Acta Cryst. E64, o772.

Marimuthu, T., Bala, M. D. \& Friedrich, H. B. (2008c). Acta Cryst. E64, o1984o1985.

Seibold, S., Schafer, A., Lohstroh, W., Walter, O. \& Doring, M. (2008). J. Appl. Polym. Sci. 108, 264-271.

Shau, M. D., Lin, C. W., Yang, W. H. \& Lin, H. R. (2002). J. Appl. Polym. Sci. 84, 950-961.

Sheldrick, G. M. (2008). Acta Cryst. A64, 112-122. 


\section{supporting information}

Acta Cryst. (2009). E65, o828 [doi:10.1107/S1600536809009817]

\section{2,8-Dimethyl-10-p-tolyl-10H-phenoxaphosphine}

\section{Thashree Marimuthu, Muhammad D. Bala and Holger B. Friedrich}

\section{S1. Comment}

The title compound was prepared as part of an ongoing study of bidentate and tridentate xanthene-based ligands (Marimuthu et al. $(2008 a, b, c)$. Similar ligands have shown relative success for the Rh-catalysed hydroformylation of alkenes. The title compound is an example of a modified xanthene backbone where a phosphorous atom has been substituted into the central ring in order to investigate the electronic properties of the final target ligand when complexed to a metal centre. In addition, methyl groups are present on the outer rings in order to increase the solubility of a resulting catalyst. The outer rings of the phenoxaphosphine backbone are nearly coplanar (dihedral angle of $\left.6.56(2)^{\circ}\right)$. This value is significantly different from the dihedral angle of $15^{\circ}$ reported by Mann et al.(1976) for 10-phenylphenoxaphosphine, which was observed to have a boat-like conformation about the $\mathrm{P}-\mathrm{O}$ axis. The $\mathrm{C} 15-\mathrm{P} 1$ bond length for the tolyl group is 1.835 (13) $\AA$, which is longer than the C-P bond lengths of the backbone heterocycle (1.805 (13) and 1.809 (13) $\AA$ for $\mathrm{C} 1-\mathrm{P} 1$ and $\mathrm{C} 12-\mathrm{P} 1$, respectively). The longer $\mathrm{C} 15-\mathrm{P} 1$ bond length is due to the pyramidal geometry at the $\mathrm{P}$ atom. Hence, the $\mathrm{C}-\mathrm{P}-\mathrm{C}$ angles range from 98.0087 (6) to $101.04(6)^{\circ}$. The ring of the toluene group is nearly perpendicular to the mean plane through the phenoxaphosphine backbone, forming a dihedral angle of $83.26(3)^{\circ}$

\section{S2. Experimental}

The synthesis of the title compound was modified from literature (Bronger et al. 2004). In an inert nitrogen atmosphere $\mathrm{AlCl}_{3}(2.5 \mathrm{~g}, 18.9 \mathrm{mmol})$ was added to $p$-tolylether $(2.5 \mathrm{~g}, 12.6 \mathrm{mmol})$ in $9 \mathrm{ml}$ phosphorous trichloride $\left(\mathrm{PCl}_{3}\right)$. The reaction mixture was refluxed for $8 \mathrm{~h}$ at $358 \mathrm{~K}$ and thereafter the excess $\mathrm{PCl}_{3}$ was distilled off at $363 \mathrm{~K}$. At this temperature, excess anhydrous toluene was added to the reaction mixture. The remaining $\mathrm{PCl}_{3}$ and toluene was distilled off at $383 \mathrm{~K}$ to afford an orange-red residue. The residue was again diluted with $15 \mathrm{ml}$ of toluene and cooled to $273 \mathrm{~K}$, followed by the dropwise addition of $3.6 \mathrm{ml}$ pyridine to the mixture while stirring. After an hour, the resulting salts were filtered off and the yellow residue extracted with toluene. The solvent was removed in vacuo, and the crude product purified by filtration through a short plug of silica gel to yield $2.45 \mathrm{~g}$ of the title compound as an oil that solidified at room temperature. X-ray quality crystals were grown from a 2-propanol/dichloromethane (1:1 v/v) solution (Yield: $61 \%$; m.p. $337-338$ K). Spectroscopic analysis: ${ }^{1} \mathrm{H}$ NMR: $\left(400 \mathrm{MHz}, \mathrm{C}_{6} \mathrm{D}_{6}, \delta\right.$, p.p.m): 1.98 (s, 6H), 1.90 (s, 3H), 6.78 (d, 2H; $\mathrm{J}(\mathrm{H}, \mathrm{H})=7.2 \mathrm{~Hz}$,), $6.85(\mathrm{dd}, 2 \mathrm{H} ; \mathrm{CH} ; \mathrm{J}(\mathrm{H}, \mathrm{H})=2.7,1.7 \mathrm{~Hz}),$,7.09 (d, 2H; J(H,H) = 8.3 Hz,), 7.30 (dd, 2H; J(H,H) = 1.9, $1.6 \mathrm{~Hz},), 7.39(\mathrm{t}, 2 \mathrm{H}, \mathrm{J}(\mathrm{H}, \mathrm{H})=7.9 \mathrm{~Hz})$. MS: $\mathrm{m} / z(\%): 357.1\left(M+\mathrm{K}^{+}\right)$calculated $=357.1$ for $\mathrm{C}_{21} \mathrm{H}_{19} \mathrm{OPK}^{+}$. FTIR: $\mathrm{cm}^{-1}=$ 3009(w), (CH), 2920(s), 1585(w), 1489(m), 1466(vs), 1385(s), 1295(s), 1265(vs), 1231(vs), 909(m).

\section{S3. Refinement}

Non-H atoms were first refined isotropically followed by anisotropic refinement by full matrix least-squares calculations based on $F^{2}$ using SHELXTL. All hydrogen atoms were first located in a difference Fourier map, then positioned geometrically and allowed to ride on their parent atoms, with $\mathrm{C}-\mathrm{H}=0.95-0.99 \AA$ and with $U_{\mathrm{iso}}(\mathrm{H})=1.2 U_{\mathrm{eq}}(\mathrm{C})$ for aryl 
$\mathrm{H}$ or $1.5 U_{\text {eq }}(\mathrm{C})$ for alkyl $\mathrm{H}$ atoms.

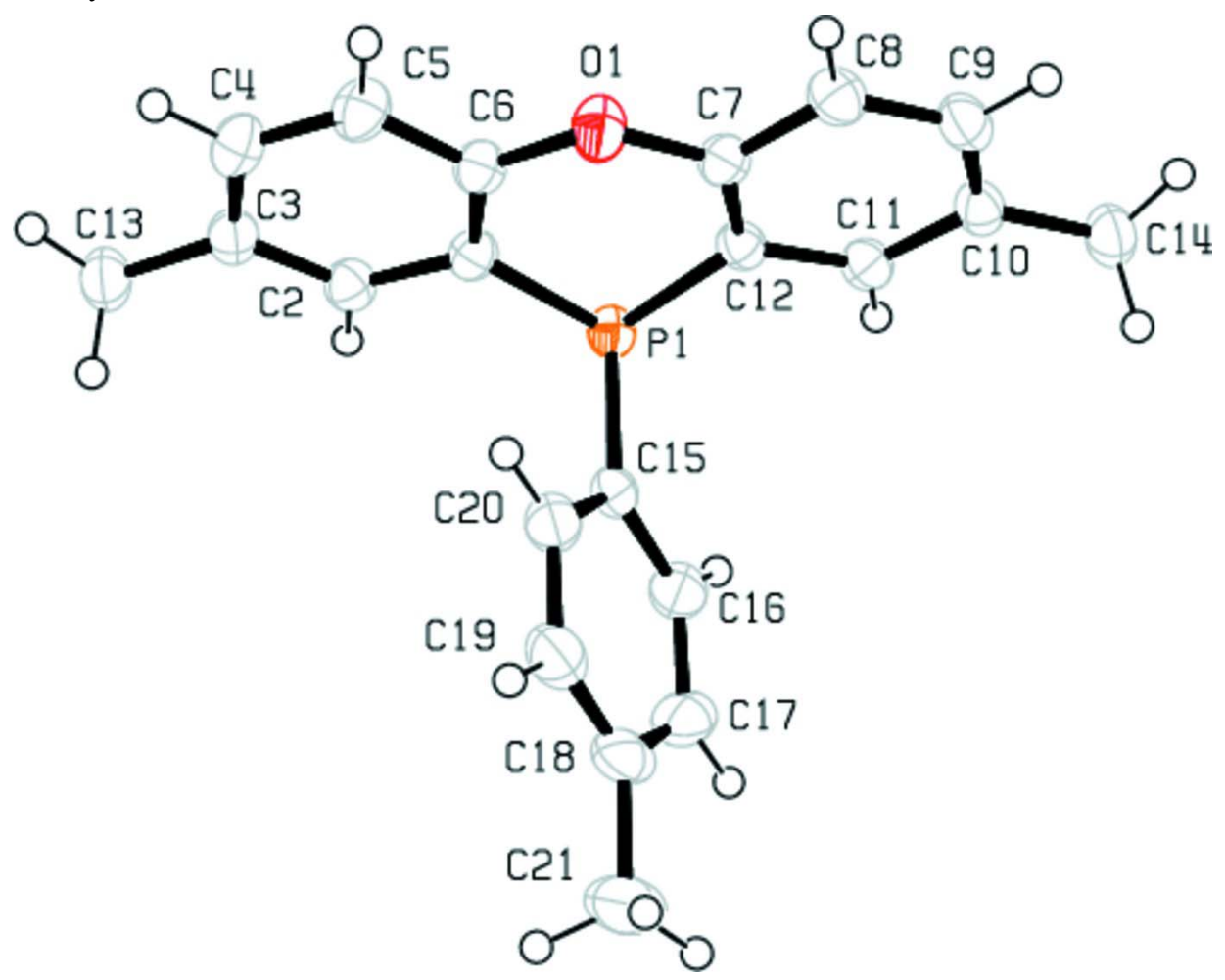

\section{Figure 1}

The molecular structure of the title compound. Thermal ellipsoids are shown at the 50\% probability level.

\section{3,6-dimethyl-10-p-tolyl-9-oxa-10-phosphaanthracene}

Crystal data

$\mathrm{C}_{21} \mathrm{H}_{19} \mathrm{OP}$

$M_{r}=318.33$

Monoclinic, $P 2_{1} / c$

Hall symbol: -P 2ybc

$a=10.9363(3) \AA$

$b=11.6323(3) \AA$

$c=14.0458$ (4) $\AA$

$\beta=111.532(1)^{\circ}$

$V=1662.13(8) \AA^{3}$

$Z=4$

Data collection

Bruker APEXII CCD area-detector diffractometer

Radiation source: fine-focus sealed tube

Graphite monochromator

$\varphi$ and $\omega$ scans

29900 measured reflections

4013 independent reflections
$F(000)=672$

$D_{\mathrm{x}}=1.272 \mathrm{Mg} \mathrm{m}^{-3}$

Mo $K \alpha$ radiation, $\lambda=0.71073 \AA$

Cell parameters from 8225 reflections

$\theta=2.3-28.4^{\circ}$

$\mu=0.17 \mathrm{~mm}^{-1}$

$T=173 \mathrm{~K}$

Needle, colourless

$0.51 \times 0.49 \times 0.48 \mathrm{~mm}$

3507 reflections with $I>2 \sigma(I)$

$R_{\text {int }}=0.046$

$\theta_{\text {max }}=28.0^{\circ}, \theta_{\min }=2.0^{\circ}$

$h=-14 \rightarrow 14$

$k=-15 \rightarrow 15$

$l=-18 \rightarrow 18$ 


\section{Refinement}

Refinement on $F^{2}$

Least-squares matrix: full

$R\left[F^{2}>2 \sigma\left(F^{2}\right)\right]=0.037$

$w R\left(F^{2}\right)=0.108$

$S=1.07$

4013 reflections

211 parameters

0 restraints

Primary atom site location: structure-invariant direct methods
Secondary atom site location: difference Fourier map

Hydrogen site location: inferred from neighbouring sites

$\mathrm{H}$-atom parameters constrained

$w=1 /\left[\sigma^{2}\left(F_{\mathrm{o}}^{2}\right)+(0.0534 P)^{2}+0.586 P\right]$ where $P=\left(F_{\mathrm{o}}{ }^{2}+2 F_{\mathrm{c}}{ }^{2}\right) / 3$

$(\Delta / \sigma)_{\max }=0.040$

$\Delta \rho_{\max }=0.37 \mathrm{e} \AA^{-3}$

$\Delta \rho_{\min }=-0.27$ e $\AA^{-3}$

Special details

Geometry. All e.s.d.'s (except the e.s.d. in the dihedral angle between two l.s. planes) are estimated using the full covariance matrix. The cell e.s.d.'s are taken into account individually in the estimation of e.s.d.'s in distances, angles and torsion angles; correlations between e.s.d.'s in cell parameters are only used when they are defined by crystal symmetry. An approximate (isotropic) treatment of cell e.s.d.'s is used for estimating e.s.d.'s involving 1.s. planes.

Refinement. Refinement of $F^{2}$ against ALL reflections. The weighted $R$-factor $w R$ and goodness of fit $S$ are based on $F^{2}$, conventional $R$-factors $R$ are based on $F$, with $F$ set to zero for negative $F^{2}$. The threshold expression of $F^{2}>\sigma\left(F^{2}\right)$ is used only for calculating $R$-factors (gt) etc. and is not relevant to the choice of reflections for refinement. $R$-factors based on $F^{2}$ are statistically about twice as large as those based on $F$, and $R$ - factors based on ALL data will be even larger.

Fractional atomic coordinates and isotropic or equivalent isotropic displacement parameters $\left(\AA^{2}\right)$

\begin{tabular}{lllll}
\hline & $x$ & $y$ & $z$ & $U_{\mathrm{iso}} * / U_{\mathrm{eq}}$ \\
\hline $\mathrm{C} 1$ & $0.59638(12)$ & $1.06591(11)$ & $0.15703(10)$ & $0.0274(3)$ \\
$\mathrm{C} 2$ & $0.53669(13)$ & $1.05047(12)$ & $0.22849(10)$ & $0.0303(3)$ \\
$\mathrm{H} 2$ & 0.5484 & 0.9791 & 0.2636 & $0.036^{*}$ \\
$\mathrm{C} 3$ & $0.46153(13)$ & $1.13388(12)$ & $0.25049(10)$ & $0.0313(3)$ \\
C4 & $0.44183(14)$ & $1.23579(12)$ & $0.19518(11)$ & $0.0361(3)$ \\
H4 & 0.3880 & 1.2940 & 0.2069 & $0.043^{*}$ \\
C5 & $0.49875(14)$ & $1.25429(12)$ & $0.12363(11)$ & $0.0357(3)$ \\
H5 & 0.4843 & 1.3247 & 0.0868 & $0.043^{*}$ \\
C6 & $0.57734(13)$ & $1.16977(11)$ & $0.10547(10)$ & $0.0289(3)$ \\
C7 & $0.69900(12)$ & $1.12246(11)$ & $-0.00204(10)$ & $0.0284(3)$ \\
C8 & $0.73269(14)$ & $1.16321(12)$ & $-0.08200(10)$ & $0.0340(3)$ \\
H8 & 0.7092 & 1.2391 & -0.1071 & $0.041^{*}$ \\
C9 & $0.80050(14)$ & $1.09313(13)$ & $-0.12506(10)$ & $0.0347(3)$ \\
H9 & 0.8246 & 1.1222 & -0.1789 & $0.042^{*}$ \\
C10 & $0.83423(13)$ & $0.98126(12)$ & $-0.09135(10)$ & $0.0310(3)$ \\
C11 & $0.79734(12)$ & $0.94259(11)$ & $-0.01249(10)$ & $0.0294(3)$ \\
H11 & 0.8177 & 0.8657 & 0.0106 & $0.035^{*}$ \\
C12 & $0.73184(12)$ & $1.01137(11)$ & $0.03436(9)$ & $0.0264(2)$ \\
C13 & $0.40629(15)$ & $1.11516(14)$ & $0.33266(11)$ & $0.0399(3)$ \\
H13A & 0.3553 & 1.0437 & 0.3190 & $0.060^{*}$ \\
H13B & 0.3491 & 1.1798 & 0.3333 & $0.060^{*}$ \\
H13C & 0.4784 & 1.1098 & 0.3993 & $0.060^{*}$ \\
C14 & $0.90781(15)$ & $0.90328(14)$ & $-0.13659(11)$ & $0.0387(3)$ \\
H14A & 0.9981 & 0.8935 & -0.0878 & $0.058^{*}$ \\
H14B & 0.9093 & 0.9371 & -0.2001 & $0.058^{*}$ \\
& & & &
\end{tabular}




$\begin{array}{lllll}\mathrm{H} 14 \mathrm{C} & 0.8641 & 0.8283 & -0.1515 & 0.058^{*} \\ \mathrm{C} 15 & 0.85437(13) & 0.98293(12) & 0.24735(9) & 0.0286(3) \\ \mathrm{C} 16 & 0.94457(15) & 0.89406(13) & 0.28148(11) & 0.0377(3) \\ \mathrm{H} 16 & 0.9249 & 0.8209 & 0.2493 & 0.045^{*} \\ \mathrm{C} 17 & 1.06328(16) & 0.91088(17) & 0.36216(12) & 0.0474(4) \\ \mathrm{H} 17 & 1.1246 & 0.8494 & 0.3837 & 0.057^{*} \\ \mathrm{C} 18 & 1.09347(15) & 1.01549(17) & 0.41141(11) & 0.0463(4) \\ \mathrm{C} 19 & 1.00354(15) & 1.10404(16) & 0.37752(11) & 0.0440(4) \\ \mathrm{H} 19 & 1.0230 & 1.1767 & 0.4106 & 0.053^{*} \\ \mathrm{C} 20 & 0.88574(14) & 1.08866(13) & 0.29636(11) & 0.0358(3) \\ \mathrm{H} 20 & 0.8258 & 1.1509 & 0.2739 & 0.043^{*} \\ \mathrm{C} 21 & 1.22049(18) & 1.0331(2) & 0.50093(13) & 0.0681(6) \\ \mathrm{H} 21 \mathrm{~A} & 1.2587 & 1.1074 & 0.4940 & 0.102^{*} \\ \mathrm{H} 21 \mathrm{~B} & 1.2821 & 0.9713 & 0.5024 & 0.102^{*} \\ \mathrm{H} 21 \mathrm{C} & 1.2033 & 1.0322 & 0.5647 & 0.102^{*} \\ \text { O1 } & 0.63326(10) & 1.20048(8) & 0.03575(8) & 0.0356(2) \\ \text { P1 } & 0.69921(3) & 0.95166(3) & 0.14160(2) & 0.02660(11) \\ \end{array}$

Atomic displacement parameters $\left(\AA^{2}\right)$

\begin{tabular}{lllllll}
\hline & $U^{11}$ & $U^{22}$ & $U^{33}$ & $U^{12}$ & $U^{13}$ & $U^{23}$ \\
\hline C1 & $0.0250(6)$ & $0.0274(6)$ & $0.0299(6)$ & $-0.0007(5)$ & $0.0102(5)$ & $-0.0010(5)$ \\
C2 & $0.0298(6)$ & $0.0307(6)$ & $0.0311(6)$ & $-0.0013(5)$ & $0.0122(5)$ & $0.0003(5)$ \\
C3 & $0.0279(6)$ & $0.0352(7)$ & $0.0316(6)$ & $-0.0036(5)$ & $0.0119(5)$ & $-0.0059(5)$ \\
C4 & $0.0349(7)$ & $0.0322(7)$ & $0.0445(8)$ & $0.0023(5)$ & $0.0185(6)$ & $-0.0054(6)$ \\
C5 & $0.0383(7)$ & $0.0273(6)$ & $0.0439(8)$ & $0.0038(5)$ & $0.0179(6)$ & $0.0021(6)$ \\
C6 & $0.0287(6)$ & $0.0280(6)$ & $0.0313(6)$ & $-0.0008(5)$ & $0.0125(5)$ & $0.0000(5)$ \\
C7 & $0.0277(6)$ & $0.0290(6)$ & $0.0288(6)$ & $-0.0008(5)$ & $0.0105(5)$ & $0.0001(5)$ \\
C8 & $0.0373(7)$ & $0.0331(7)$ & $0.0332(7)$ & $-0.0006(5)$ & $0.0147(6)$ & $0.0059(5)$ \\
C9 & $0.0370(7)$ & $0.0418(8)$ & $0.0279(6)$ & $-0.0051(6)$ & $0.0150(5)$ & $0.0011(5)$ \\
C10 & $0.0295(6)$ & $0.0372(7)$ & $0.0267(6)$ & $-0.0050(5)$ & $0.0110(5)$ & $-0.0063(5)$ \\
C11 & $0.0308(6)$ & $0.0289(6)$ & $0.0279(6)$ & $-0.0016(5)$ & $0.0100(5)$ & $-0.0026(5)$ \\
C12 & $0.0263(6)$ & $0.0285(6)$ & $0.0239(5)$ & $-0.0034(5)$ & $0.0084(5)$ & $-0.0013(5)$ \\
C13 & $0.0402(8)$ & $0.0469(9)$ & $0.0385(7)$ & $0.0005(6)$ & $0.0214(6)$ & $-0.0047(6)$ \\
C14 & $0.0404(8)$ & $0.0456(8)$ & $0.0346(7)$ & $-0.0017(6)$ & $0.0190(6)$ & $-0.0073(6)$ \\
C15 & $0.0304(6)$ & $0.0337(6)$ & $0.0250(6)$ & $0.0017(5)$ & $0.0140(5)$ & $0.0048(5)$ \\
C16 & $0.0413(8)$ & $0.0374(7)$ & $0.0366(7)$ & $0.0062(6)$ & $0.0168(6)$ & $0.0111(6)$ \\
C17 & $0.0379(8)$ & $0.0626(10)$ & $0.0412(8)$ & $0.0109(7)$ & $0.0140(7)$ & $0.0220(8)$ \\
C18 & $0.0342(7)$ & $0.0774(12)$ & $0.0272(7)$ & $-0.0079(8)$ & $0.0111(6)$ & $0.0112(7)$ \\
C19 & $0.0416(8)$ & $0.0588(10)$ & $0.0334(7)$ & $-0.0115(7)$ & $0.0159(6)$ & $-0.0083(7)$ \\
C20 & $0.0350(7)$ & $0.0398(7)$ & $0.0338(7)$ & $-0.0013(6)$ & $0.0141(6)$ & $-0.0030(6)$ \\
C21 & $0.0400(9)$ & $0.1219(19)$ & $0.0356(8)$ & $-0.0161(10)$ & $0.0060(7)$ & $0.0136(10)$ \\
O1 & $0.0444(6)$ & $0.0277(5)$ & $0.0431(5)$ & $0.0054(4)$ & $0.0261(5)$ & $0.0070(4)$ \\
P1 & $0.02996(18)$ & $0.02340(17)$ & $0.02918(18)$ & $0.00000(12)$ & $0.01410(14)$ & $0.00138(12)$ \\
& & & & & &
\end{tabular}


Geometric parameters $\left(\AA,{ }^{\circ}\right)$

\begin{tabular}{|c|c|c|c|}
\hline $\mathrm{C} 1-\mathrm{C} 6$ & $1.3845(18)$ & $\mathrm{C} 12-\mathrm{P} 1$ & $1.8092(13)$ \\
\hline $\mathrm{C} 1-\mathrm{C} 2$ & $1.3953(18)$ & $\mathrm{C} 13-\mathrm{H} 13 \mathrm{~A}$ & 0.9800 \\
\hline $\mathrm{C} 1-\mathrm{P} 1$ & $1.8046(13)$ & $\mathrm{C} 13-\mathrm{H} 13 \mathrm{~B}$ & 0.9800 \\
\hline $\mathrm{C} 2-\mathrm{C} 3$ & $1.3780(18)$ & $\mathrm{C} 13-\mathrm{H} 13 \mathrm{C}$ & 0.9800 \\
\hline $\mathrm{C} 2-\mathrm{H} 2$ & 0.9500 & $\mathrm{C} 14-\mathrm{H} 14 \mathrm{~A}$ & 0.9800 \\
\hline $\mathrm{C} 3-\mathrm{C} 4$ & $1.390(2)$ & $\mathrm{C} 14-\mathrm{H} 14 \mathrm{~B}$ & 0.9800 \\
\hline $\mathrm{C} 3-\mathrm{C} 13$ & $1.5021(19)$ & $\mathrm{C} 14-\mathrm{H} 14 \mathrm{C}$ & 0.9800 \\
\hline $\mathrm{C} 4-\mathrm{C} 5$ & $1.379(2)$ & $\mathrm{C} 15-\mathrm{C} 16$ & $1.3873(19)$ \\
\hline $\mathrm{C} 4-\mathrm{H} 4$ & 0.9500 & $\mathrm{C} 15-\mathrm{C} 20$ & $1.390(2)$ \\
\hline $\mathrm{C} 5-\mathrm{C} 6$ & $1.3896(19)$ & $\mathrm{C} 15-\mathrm{P} 1$ & $1.8352(13)$ \\
\hline $\mathrm{C} 5-\mathrm{H} 5$ & 0.9500 & $\mathrm{C} 16-\mathrm{C} 17$ & $1.389(2)$ \\
\hline $\mathrm{C} 6-\mathrm{O} 1$ & $1.3784(15)$ & $\mathrm{C} 16-\mathrm{H} 16$ & 0.9500 \\
\hline $\mathrm{C} 7-\mathrm{O} 1$ & $1.3794(16)$ & $\mathrm{C} 17-\mathrm{C} 18$ & $1.379(3)$ \\
\hline $\mathrm{C} 7-\mathrm{C} 8$ & $1.3876(18)$ & $\mathrm{C} 17-\mathrm{H} 17$ & 0.9500 \\
\hline $\mathrm{C} 7-\mathrm{C} 12$ & $1.3876(18)$ & $\mathrm{C} 18-\mathrm{C} 19$ & $1.382(3)$ \\
\hline $\mathrm{C} 8-\mathrm{C} 9$ & $1.382(2)$ & $\mathrm{C} 18-\mathrm{C} 21$ & $1.507(2)$ \\
\hline $\mathrm{C} 8-\mathrm{H} 8$ & 0.9500 & $\mathrm{C} 19-\mathrm{C} 20$ & $1.383(2)$ \\
\hline $\mathrm{C} 9-\mathrm{C} 10$ & $1.388(2)$ & C19-H19 & 0.9500 \\
\hline $\mathrm{C} 9-\mathrm{H} 9$ & 0.9500 & $\mathrm{C} 20-\mathrm{H} 20$ & 0.9500 \\
\hline $\mathrm{C} 10-\mathrm{C} 11$ & $1.3858(18)$ & $\mathrm{C} 21-\mathrm{H} 21 \mathrm{~A}$ & 0.9800 \\
\hline $\mathrm{C} 10-\mathrm{C} 14$ & $1.4999(19)$ & $\mathrm{C} 21-\mathrm{H} 21 \mathrm{~B}$ & 0.9800 \\
\hline $\mathrm{C} 11-\mathrm{C} 12$ & $1.3901(18)$ & $\mathrm{C} 21-\mathrm{H} 21 \mathrm{C}$ & 0.9800 \\
\hline $\mathrm{C} 11-\mathrm{H} 11$ & 0.9500 & & \\
\hline $\mathrm{C} 6-\mathrm{C} 1-\mathrm{C} 2$ & $117.93(12)$ & $\mathrm{H} 13 \mathrm{~A}-\mathrm{C} 13-\mathrm{H} 13 \mathrm{~B}$ & 109.5 \\
\hline $\mathrm{C} 6-\mathrm{C} 1-\mathrm{P} 1$ & $124.10(10)$ & $\mathrm{C} 3-\mathrm{C} 13-\mathrm{H} 13 \mathrm{C}$ & 109.5 \\
\hline $\mathrm{C} 2-\mathrm{C} 1-\mathrm{P} 1$ & $117.92(10)$ & $\mathrm{H} 13 \mathrm{~A}-\mathrm{C} 13-\mathrm{H} 13 \mathrm{C}$ & 109.5 \\
\hline $\mathrm{C} 3-\mathrm{C} 2-\mathrm{C} 1$ & $123.08(12)$ & $\mathrm{H} 13 \mathrm{~B}-\mathrm{C} 13-\mathrm{H} 13 \mathrm{C}$ & 109.5 \\
\hline $\mathrm{C} 3-\mathrm{C} 2-\mathrm{H} 2$ & 118.5 & $\mathrm{C} 10-\mathrm{C} 14-\mathrm{H} 14 \mathrm{~A}$ & 109.5 \\
\hline $\mathrm{C} 1-\mathrm{C} 2-\mathrm{H} 2$ & 118.5 & $\mathrm{C} 10-\mathrm{C} 14-\mathrm{H} 14 \mathrm{~B}$ & 109.5 \\
\hline $\mathrm{C} 2-\mathrm{C} 3-\mathrm{C} 4$ & $117.25(12)$ & $\mathrm{H} 14 \mathrm{~A}-\mathrm{C} 14-\mathrm{H} 14 \mathrm{~B}$ & 109.5 \\
\hline $\mathrm{C} 2-\mathrm{C} 3-\mathrm{C} 13$ & $120.76(13)$ & $\mathrm{C} 10-\mathrm{C} 14-\mathrm{H} 14 \mathrm{C}$ & 109.5 \\
\hline $\mathrm{C} 4-\mathrm{C} 3-\mathrm{C} 13$ & $121.98(13)$ & $\mathrm{H} 14 \mathrm{~A}-\mathrm{C} 14-\mathrm{H} 14 \mathrm{C}$ & 109.5 \\
\hline $\mathrm{C} 5-\mathrm{C} 4-\mathrm{C} 3$ & $121.42(13)$ & $\mathrm{H} 14 \mathrm{~B}-\mathrm{C} 14-\mathrm{H} 14 \mathrm{C}$ & 109.5 \\
\hline $\mathrm{C} 5-\mathrm{C} 4-\mathrm{H} 4$ & 119.3 & $\mathrm{C} 16-\mathrm{C} 15-\mathrm{C} 20$ & $118.26(13)$ \\
\hline $\mathrm{C} 3-\mathrm{C} 4-\mathrm{H} 4$ & 119.3 & $\mathrm{C} 16-\mathrm{C} 15-\mathrm{P} 1$ & $117.45(11)$ \\
\hline $\mathrm{C} 4-\mathrm{C} 5-\mathrm{C} 6$ & $119.89(13)$ & $\mathrm{C} 20-\mathrm{C} 15-\mathrm{P} 1$ & $124.28(11)$ \\
\hline $\mathrm{C} 4-\mathrm{C} 5-\mathrm{H} 5$ & 120.1 & $\mathrm{C} 15-\mathrm{C} 16-\mathrm{C} 17$ & $120.68(15)$ \\
\hline $\mathrm{C} 6-\mathrm{C} 5-\mathrm{H} 5$ & 120.1 & $\mathrm{C} 15-\mathrm{C} 16-\mathrm{H} 16$ & 119.7 \\
\hline $\mathrm{O} 1-\mathrm{C} 6-\mathrm{C} 1$ & $125.24(12)$ & $\mathrm{C} 17-\mathrm{C} 16-\mathrm{H} 16$ & 119.7 \\
\hline $\mathrm{O} 1-\mathrm{C} 6-\mathrm{C} 5$ & $114.37(12)$ & $\mathrm{C} 18-\mathrm{C} 17-\mathrm{C} 16$ & $120.93(15)$ \\
\hline $\mathrm{C} 1-\mathrm{C} 6-\mathrm{C} 5$ & $120.38(12)$ & $\mathrm{C} 18-\mathrm{C} 17-\mathrm{H} 17$ & 119.5 \\
\hline $\mathrm{O} 1-\mathrm{C} 7-\mathrm{C} 8$ & $114.67(12)$ & $\mathrm{C} 16-\mathrm{C} 17-\mathrm{H} 17$ & 119.5 \\
\hline $\mathrm{O} 1-\mathrm{C} 7-\mathrm{C} 12$ & $124.88(11)$ & $\mathrm{C} 17-\mathrm{C} 18-\mathrm{C} 19$ & $118.41(14)$ \\
\hline $\mathrm{C} 8-\mathrm{C} 7-\mathrm{C} 12$ & $120.45(12)$ & $\mathrm{C} 17-\mathrm{C} 18-\mathrm{C} 21$ & $121.11(18)$ \\
\hline $\mathrm{C} 9-\mathrm{C} 8-\mathrm{C} 7$ & $119.82(13)$ & $\mathrm{C} 19-\mathrm{C} 18-\mathrm{C} 21$ & $120.48(19)$ \\
\hline
\end{tabular}




\begin{tabular}{|c|c|c|c|}
\hline $\mathrm{C} 9-\mathrm{C} 8-\mathrm{H} 8$ & 120.1 & $\mathrm{C} 18-\mathrm{C} 19-\mathrm{C} 20$ & $121.14(16)$ \\
\hline $\mathrm{C} 7-\mathrm{C} 8-\mathrm{H} 8$ & 120.1 & $\mathrm{C} 18-\mathrm{C} 19-\mathrm{H} 19$ & 119.4 \\
\hline $\mathrm{C} 8-\mathrm{C} 9-\mathrm{C} 10$ & $121.47(12)$ & $\mathrm{C} 20-\mathrm{C} 19-\mathrm{H} 19$ & 119.4 \\
\hline $\mathrm{C} 8-\mathrm{C} 9-\mathrm{H} 9$ & 119.3 & $\mathrm{C} 19-\mathrm{C} 20-\mathrm{C} 15$ & $120.58(15)$ \\
\hline $\mathrm{C} 10-\mathrm{C} 9-\mathrm{H} 9$ & 119.3 & $\mathrm{C} 19-\mathrm{C} 20-\mathrm{H} 20$ & 119.7 \\
\hline $\mathrm{C} 11-\mathrm{C} 10-\mathrm{C} 9$ & $117.27(12)$ & $\mathrm{C} 15-\mathrm{C} 20-\mathrm{H} 20$ & 119.7 \\
\hline $\mathrm{C} 11-\mathrm{C} 10-\mathrm{C} 14$ & $120.08(13)$ & $\mathrm{C} 18-\mathrm{C} 21-\mathrm{H} 21 \mathrm{~A}$ & 109.5 \\
\hline $\mathrm{C} 9-\mathrm{C} 10-\mathrm{C} 14$ & $122.64(12)$ & $\mathrm{C} 18-\mathrm{C} 21-\mathrm{H} 21 \mathrm{~B}$ & 109.5 \\
\hline $\mathrm{C} 10-\mathrm{C} 11-\mathrm{C} 12$ & $122.92(12)$ & $\mathrm{H} 21 \mathrm{~A}-\mathrm{C} 21-\mathrm{H} 21 \mathrm{~B}$ & 109.5 \\
\hline $\mathrm{C} 10-\mathrm{C} 11-\mathrm{H} 11$ & 118.5 & $\mathrm{C} 18-\mathrm{C} 21-\mathrm{H} 21 \mathrm{C}$ & 109.5 \\
\hline $\mathrm{C} 12-\mathrm{C} 11-\mathrm{H} 11$ & 118.5 & $\mathrm{H} 21 \mathrm{~A}-\mathrm{C} 21-\mathrm{H} 21 \mathrm{C}$ & 109.5 \\
\hline $\mathrm{C} 7-\mathrm{C} 12-\mathrm{C} 11$ & $118.04(12)$ & $\mathrm{H} 21 \mathrm{~B}-\mathrm{C} 21-\mathrm{H} 21 \mathrm{C}$ & 109.5 \\
\hline $\mathrm{C} 7-\mathrm{C} 12-\mathrm{P} 1$ & $124.12(10)$ & $\mathrm{C} 6-\mathrm{O} 1-\mathrm{C} 7$ & $122.19(10)$ \\
\hline $\mathrm{C} 11-\mathrm{C} 12-\mathrm{P} 1$ & $117.81(10)$ & $\mathrm{C} 1-\mathrm{P} 1-\mathrm{C} 12$ & $98.00(6)$ \\
\hline $\mathrm{C} 3-\mathrm{C} 13-\mathrm{H} 13 \mathrm{~A}$ & 109.5 & $\mathrm{C} 1-\mathrm{P} 1-\mathrm{C} 15$ & $100.87(6)$ \\
\hline $\mathrm{C} 3-\mathrm{C} 13-\mathrm{H} 13 \mathrm{~B}$ & 109.5 & $\mathrm{C} 12-\mathrm{P} 1-\mathrm{C} 15$ & $101.04(6)$ \\
\hline $\mathrm{C} 6-\mathrm{C} 1-\mathrm{C} 2-\mathrm{C} 3$ & $-0.7(2)$ & $\mathrm{C} 20-\mathrm{C} 15-\mathrm{C} 16-\mathrm{C} 17$ & $-0.3(2)$ \\
\hline $\mathrm{P} 1-\mathrm{C} 1-\mathrm{C} 2-\mathrm{C} 3$ & $176.68(10)$ & $\mathrm{P} 1-\mathrm{C} 15-\mathrm{C} 16-\mathrm{C} 17$ & $-179.07(11)$ \\
\hline $\mathrm{C} 1-\mathrm{C} 2-\mathrm{C} 3-\mathrm{C} 4$ & $2.4(2)$ & $\mathrm{C} 15-\mathrm{C} 16-\mathrm{C} 17-\mathrm{C} 18$ & $1.1(2)$ \\
\hline $\mathrm{C} 1-\mathrm{C} 2-\mathrm{C} 3-\mathrm{C} 13$ & $-176.31(13)$ & $\mathrm{C} 16-\mathrm{C} 17-\mathrm{C} 18-\mathrm{C} 19$ & $-1.0(2)$ \\
\hline $\mathrm{C} 2-\mathrm{C} 3-\mathrm{C} 4-\mathrm{C} 5$ & $-2.1(2)$ & $\mathrm{C} 16-\mathrm{C} 17-\mathrm{C} 18-\mathrm{C} 21$ & $178.23(14)$ \\
\hline $\mathrm{C} 13-\mathrm{C} 3-\mathrm{C} 4-\mathrm{C} 5$ & $176.57(13)$ & $\mathrm{C} 17-\mathrm{C} 18-\mathrm{C} 19-\mathrm{C} 20$ & $0.1(2)$ \\
\hline $\mathrm{C} 3-\mathrm{C} 4-\mathrm{C} 5-\mathrm{C} 6$ & $0.2(2)$ & $\mathrm{C} 21-\mathrm{C} 18-\mathrm{C} 19-\mathrm{C} 20$ & $-179.10(14)$ \\
\hline $\mathrm{C} 2-\mathrm{C} 1-\mathrm{C} 6-\mathrm{O} 1$ & $177.32(12)$ & $\mathrm{C} 18-\mathrm{C} 19-\mathrm{C} 20-\mathrm{C} 15$ & $0.6(2)$ \\
\hline $\mathrm{P} 1-\mathrm{C} 1-\mathrm{C} 6-\mathrm{O} 1$ & $0.09(19)$ & $\mathrm{C} 16-\mathrm{C} 15-\mathrm{C} 20-\mathrm{C} 19$ & $-0.5(2)$ \\
\hline $\mathrm{C} 2-\mathrm{C} 1-\mathrm{C} 6-\mathrm{C} 5$ & $-1.29(19)$ & $\mathrm{P} 1-\mathrm{C} 15-\mathrm{C} 20-\mathrm{C} 19$ & $178.12(11)$ \\
\hline $\mathrm{P} 1-\mathrm{C} 1-\mathrm{C} 6-\mathrm{C} 5$ & $-178.52(10)$ & $\mathrm{C} 1-\mathrm{C} 6-\mathrm{O} 1-\mathrm{C} 7$ & $10.4(2)$ \\
\hline $\mathrm{C} 4-\mathrm{C} 5-\mathrm{C} 6-\mathrm{O} 1$ & $-177.21(13)$ & $\mathrm{C} 5-\mathrm{C} 6-\mathrm{O} 1-\mathrm{C} 7$ & $-170.89(12)$ \\
\hline $\mathrm{C} 4-\mathrm{C} 5-\mathrm{C} 6-\mathrm{C} 1$ & $1.5(2)$ & $\mathrm{C} 8-\mathrm{C} 7-\mathrm{O} 1-\mathrm{C} 6$ & $171.17(12)$ \\
\hline $\mathrm{O} 1-\mathrm{C} 7-\mathrm{C} 8-\mathrm{C} 9$ & $179.10(12)$ & $\mathrm{C} 12-\mathrm{C} 7-\mathrm{O} 1-\mathrm{C} 6$ & $-9.1(2)$ \\
\hline $\mathrm{C} 12-\mathrm{C} 7-\mathrm{C} 8-\mathrm{C} 9$ & $-0.6(2)$ & $\mathrm{C} 6-\mathrm{C} 1-\mathrm{P} 1-\mathrm{C} 12$ & $-8.60(12)$ \\
\hline $\mathrm{C} 7-\mathrm{C} 8-\mathrm{C} 9-\mathrm{C} 10$ & $1.1(2)$ & $\mathrm{C} 2-\mathrm{C} 1-\mathrm{P} 1-\mathrm{C} 12$ & $174.17(10)$ \\
\hline $\mathrm{C} 8-\mathrm{C} 9-\mathrm{C} 10-\mathrm{C} 11$ & $-0.1(2)$ & $\mathrm{C} 6-\mathrm{C} 1-\mathrm{P} 1-\mathrm{C} 15$ & $94.34(12)$ \\
\hline $\mathrm{C} 8-\mathrm{C} 9-\mathrm{C} 10-\mathrm{C} 14$ & $-179.98(13)$ & $\mathrm{C} 2-\mathrm{C} 1-\mathrm{P} 1-\mathrm{C} 15$ & $-82.90(11)$ \\
\hline $\mathrm{C} 9-\mathrm{C} 10-\mathrm{C} 11-\mathrm{C} 12$ & $-1.45(19)$ & $\mathrm{C} 7-\mathrm{C} 12-\mathrm{P} 1-\mathrm{C} 1$ & $9.72(12)$ \\
\hline $\mathrm{C} 14-\mathrm{C} 10-\mathrm{C} 11-\mathrm{C} 12$ & $178.41(12)$ & $\mathrm{C} 11-\mathrm{C} 12-\mathrm{P} 1-\mathrm{C} 1$ & $-172.23(10)$ \\
\hline $\mathrm{O} 1-\mathrm{C} 7-\mathrm{C} 12-\mathrm{C} 11$ & $179.45(12)$ & $\mathrm{C} 7-\mathrm{C} 12-\mathrm{P} 1-\mathrm{C} 15$ & $-93.08(12)$ \\
\hline $\mathrm{C} 8-\mathrm{C} 7-\mathrm{C} 12-\mathrm{C} 11$ & $-0.86(19)$ & $\mathrm{C} 11-\mathrm{C} 12-\mathrm{P} 1-\mathrm{C} 15$ & $84.97(11)$ \\
\hline $\mathrm{O} 1-\mathrm{C} 7-\mathrm{C} 12-\mathrm{P} 1$ & $-2.50(19)$ & $\mathrm{C} 16-\mathrm{C} 15-\mathrm{P} 1-\mathrm{C} 1$ & $159.40(10)$ \\
\hline $\mathrm{C} 8-\mathrm{C} 7-\mathrm{C} 12-\mathrm{P} 1$ & $177.19(10)$ & $\mathrm{C} 20-\mathrm{C} 15-\mathrm{P} 1-\mathrm{C} 1$ & $-19.24(12)$ \\
\hline $\mathrm{C} 10-\mathrm{C} 11-\mathrm{C} 12-\mathrm{C} 7$ & $1.94(19)$ & $\mathrm{C} 16-\mathrm{C} 15-\mathrm{P} 1-\mathrm{C} 12$ & $-100.12(11)$ \\
\hline $\mathrm{C} 10-\mathrm{C} 11-\mathrm{C} 12-\mathrm{P} 1$ & $-176.23(10)$ & $\mathrm{C} 20-\mathrm{C} 15-\mathrm{P} 1-\mathrm{C} 12$ & $81.24(12)$ \\
\hline
\end{tabular}

